\title{
A New Fractal-Based Miniaturized Dual Band Patch Antenna for RF Energy Harvesting
}

\author{
Sika Shrestha, ${ }^{1}$ Seong Ro Lee, ${ }^{2}$ and Dong-You Choi ${ }^{1}$ \\ ${ }^{1}$ Department of Information and Communication Engineering, Chosun University, 309 Pilmun-daero Dong-gu, \\ Gwangju 501-759, Republic of Korea \\ ${ }^{2}$ Department of Information and Electronics Engineering, Mokpo National University, 1666 Youngsan-ro, \\ Cheonggye-myeon, Muan-gun, Jeonnam 534-729, Republic of Korea
}

Correspondence should be addressed to Dong-You Choi; dychoi@chosun.ac.kr

Received 12 September 2013; Revised 26 November 2013; Accepted 2 December 2013; Published 29 January 2014

Academic Editor: Apostolos Georgiadis

Copyright (C) 2014 Sika Shrestha et al. This is an open access article distributed under the Creative Commons Attribution License, which permits unrestricted use, distribution, and reproduction in any medium, provided the original work is properly cited.

The growth of wireless communications in recent years has made it necessary to develop compact, lightweight multiband antennas. Compact antennas can achieve the same performance as large antennas do with low price and with greater system integration. Dualfrequency microstrip antennas for transmission and reception represent promising approach for doubling the system capacity. In this work, a miniaturized dual band antenna operable at 2.45 and $5.8 \mathrm{GHz}$ is constructed by modifying the standard microstrip patch antenna geometry into a fractal structure. In addition to miniaturization and dual band nature, the proposed antenna also removes unwanted harmonics without the use of additional filter component. Using a finite-element-method-based high frequency structure simulator (HFSS), the antenna is designed and its performance in terms of return loss, impedance matching, radiation pattern, and voltage standing wave ratio (VSWR) is demonstrated. Simulation results are shown to be in close agreement with performance measurements from an actual antenna fabricated on an FR4 substrate. The proposed antenna can be integrated with a rectifier circuit to develop a compact rectenna that can harvest RF energy in both of these frequency bands at a reduction in size of $25.98 \%$ relative to a conventional rectangular patch antenna.

\section{Introduction}

In recent decades, energy harvesting from external sources such as solar radiation, thermal energy, wind, and radio frequency (RF) emissions has emerged as a promising source of environmentally friendly energy that can provide an alternative to existing resources. As energy harvesting generally uses inexhaustible sources, it can in many cases provide unlimited energy for the lifespan of an electronic device. Rectennas can be used to convert wireless RF power into utilizable DC power. Typically, the antenna and rectifier circuits in a rectenna are designed separately, with the antenna designed first using electromagnetic (EM) simulation and its parameters and then incorporated into a nonlinear circuit simulation tool used to design the rectifier. While miniaturization of a rectenna application requires that the size of its antenna be reduced as much as possible, a multiband structure that can harvest RF energy at more than one frequency would improve the energy harvesting functionality. Correspondingly, this work focuses on the design of a compact antenna for a miniaturized rectenna that can operate at two frequencies. As it uses a single wireless transceiver to support multiband operation, this multiband reduced-size antenna occupies less space, which in turn expands mobility options and simplifies installation.

Microstrip patch antennas (MPAs) are gaining popularity for use in rectenna applications owing to their advantages in terms of simplicity, lightweight, low manufacturing cost, and ability to easily integrate into feed networks. In addition, antenna patch shapes and modes can be easily chosen to match resonant frequency, polarization, signal pattern, and impedance. Several design variants exist for fabricating smallsized multiband patch antennas that can be used for RF energy harvesting [1].

Recently, the use of fractal geometry has been proposed as a method for miniaturizing standard multiband antenna 
architectures. Fractals are the class of geometrical shapes composed of multiple iterations of a single elementary shape [2], and their application can be found in various fields, including image compression algorithms, weather prediction, integrated circuits, filter design, and, most relevant to this work, fractal electrodynamics, in which fractal geometry is combined with electromagnetic theory in order to explore new classes of radiation, propagation, and scattering problems. Antenna theory and design have become one of the most promising areas of fractal electrodynamics research, and fractal antennas, which have been developed over the past 20 years, have proven to be the biggest fundamental breakthrough in antenna technology of the last half century.

A fractal element antenna is shaped using recursive nature of fractal geometry. Fractal antenna can produce fractal versions of all existing antenna types, including dipole, monopole, patch, conformal, spiral, and helical, as well as compact variants of each, possible through fractal technology. Fractal patch antennas can be designed in a number of shapes, including Sierpinski gaskets [3], Sierpinski carpets [4], Minkowski loops [5], and Koch Islands [6]. In terms of antenna design, the fractal characteristics of selfsimilarity/self-affinity and space-filling are of particular interest; the self-similarity and self-affinity properties of a fractal can be relatively understood as copies of the entire structure within the same structure at different scales. An image is reduced by the same factor in all directions for self-similarity; however, a self-affine fractal has a different scale factor for different directions. This unique feature of fractal geometry can provide additional flexibility in the antenna design, since, by selecting the scale factors appropriately, resonances can be spaced by different factors. The self-similar/self-affine properties of fractals such as the Sierpinski gasket [3] are thus seen to be useful in designing multi-frequency antennas.

As fractals constitute space-filling contours, electrically large features can be efficiently packed into small areas. Because the electrical length plays such an important role in antenna design, this packing efficiency is useful in developing viable miniaturization techniques. Using fractal geometries such as the Sierpinski carpet [4] in antenna design allows for reduction in metallization that enables current path lengthening; this in turn reduces the resonant frequency and therefore decreases the overall dimensions needed to resonate at the required frequency.

Miniaturized dual band antennas are generally complex structures that are often challenging to design. The proposed approach avoids the complexity of conventional designs, presenting instead a simple and effective approach to designing a multiband patch antenna at significantly reduced size. In this study, the second iteration of an inset-fed modified Sierpinski carpet containing perturbation and a small stub was etched onto a rectangular patch antenna in order to obtain a miniaturized dual band fractal patch antenna. The proposed antenna can operate at the unlicensed ISM (industrial, science, and medicine) central band frequencies of 2.45 and $5.8 \mathrm{GHz}$.

The remainder of the paper is organized as follows. Section 1 summarizes the various techniques adopted in previous research to obtain miniaturization and dual band functionality in patch and fractal antennas. Section 3.1 illustrates the fundamental concepts implemented in the proposed antenna design. A simulation procedure and its iterative design approach are demonstrated in Section 3.2. Simulation and fabrication results are discussed in Section 4. Finally, conclusions are provided in Section 5.

\section{Related Works}

Compact antennas represent an inevitable design step in accommodating the rapid growth of wireless applications. A number of methods have been suggested to reduce the size of microstrip antennas, including the use of high dielectric constant substrates [7], modification of basic patch shapes, short-circuiting patches to the ground plane, the use of superstrates [8], and several techniques combining these methods. The size of a circular microstrip disk antenna can be reduced by introducing slots into its surface; Yo et al. [9] were able to reduce the radius of a rectenna from 16.5 to $15.5 \mathrm{~mm}$, yielding a $12 \%$ decrease in area. Etching a cross-shaped slot onto the surface of a square aperture coupled patch antenna enabled an area reduction of $32.5 \%$ in a rectenna [10], while etching forty slits onto the perimeter of a two-port, meandered, square patch antenna (ten per side) yielded a $48 \%$ reduction in size [11]. The introduction of slits disturbs surface current flow, which in turn forces current meandering and, correspondingly, increases the electrical length of the patch antenna along both of its dimensions. The inclusion of two orthogonal pairs of irregular and asymmetric slits was proposed as a method to reduce the size of a square patch to $40 \%$ [12], and an antenna composed of the interconnection of four corner patches alternating with four strips and a fifth central patch was able to attain a surface area reduction of $60 \%$ [13].

The use of fractal geometry departs from these techniques by introducing a "non-Euclidean" method for reducing the size of a patch antenna. The purpose of using nonstandard geometry in an antenna is to increase its overall electrical length in order to lengthen its surface current paths and cause a shift in resonant frequency. Thus, by reducing the dimensions of the patch, it is possible to design a much more compact antenna for a given resonant frequency. Various fractal design integrations have been proposed to do this, including a microstrip antenna with an RO4003 substrate and Koch-shaped fractal defects on its patch surface [14] as well as a miniaturized microstrip antenna in which Sierpinski carpets are inserted into a single patch and the inner and outer edge are etched with Koch curves [15]. Jamshidifar et al. [16] introduced a miniaturized fractal butterfly patch antenna, and Shrestha et al. [4] presented an edge-fed, Sierpinski fractal-based patch antenna of significantly reduced size.

Traditional antenna structures operate within a single frequency band, with additional applications requiring the addition of new antennas. As such architecture limits available space and poses sitting problems, it is beneficial to use multiband antennas operating over many frequency bands in order to enable the simultaneous transmission of video, voice, and data information via a single antenna. Several techniques and approaches for implementing multiband antennas have been proposed, including frequency tuning by rotating a 
circular patch containing four distinct shapes, each of which corresponds to a different set of resonant frequencies [17]. A triangular, truncated-corner short-circuited antenna with a $\mathrm{V}$-shaped slot for dual band operation (i.e., in the 2.5-2.55 and 3.4-3.7 GHz WiMax bands) was proposed by Singh et al. [18], while Ali et al. [19] demonstrated that a square patch antenna containing two rectangular slots properly positioned along its diagonal can function as a dual band antenna in a rectenna assembly. Frequency diversity can be achieved by controlling the electrical length through the switching of pin diodes on the U-slot of a corner-truncated square patch antenna [20].

The self-similarity/self-affinity characteristics of fractal geometry can also be applied in the design of multiband antennas. A square microstrip patch antenna embedded with modified Sierpinski gasket slots allowed for enhanced miniaturization of a dual-frequency system [21], while a dual wideband, coplanar waveguide (CPW) fed modified Koch fractal printed-slot antenna containing a simple tuning slot that is suitable for WLAN and WiMax operation was proposed by Krishna et al. [22]. A novel dual band fractalshaped microstrip antenna for RFID applications designed to cover ISM band frequencies $(2.45$ and $5.80 \mathrm{GHz})$ was presented by Ghalibafan and Kashani [23]. Finally, Bisht and Kumar [24] demonstrated that a circular patch antenna with fractal patterns can produce dual band operation for C-band applications.

\section{Antenna Design}

Current wireless applications have challenged antenna designers with demands for low cost, high performance, easily fabricated compact multiband antennas with simple radiating elements, and signal feeding configurations. These results can be obtained by designing a modified, inset-fed Sierpinski carpet patch antenna.

\subsection{Fundamental Concepts}

3.1.1. Design Specifications. The three essential parameters for the design of a patch antenna are the resonant frequency, $f_{r}$; the dielectric constant of the substrate, $\varepsilon_{r}$; and the thickness of the substrate, $h$. Dual design frequencies of 2.45 and $5.8 \mathrm{GHz}$ are chosen here because they require low-cost components, are located in the ISM band, and experience extremely low attenuation through the atmosphere. The proposed antenna fulfils the requirements of the 2.45 and $5.8 \mathrm{GHz}$ ISM bands for RFID and other wireless applications. The design specifications of the fractal patch antenna are listed in Table 1.

\subsubsection{Rectangular Patch Design. A rectangular microstrip} patch antenna is taken as the generator of the fractal antenna. The most popular models for the analysis of microstrip patch antennas are the transmission line model, cavity model, and full-wave model (which include primarily integral equations/moment method). As the transmission line model is the simplest of these and provides a good deal of physical insight, it is adopted as the design method for the proposed antenna. In essence, the transmission line model represents a microstrip antenna as two slots, each of width $W$ and
TABle 1: Design specifications.

\begin{tabular}{lc}
\hline Antenna & Fractal patch antenna \\
\hline Substrate & FR4 \\
Relative permittivity & 4.7 \\
Dielectric loss tangent & 0.019 \\
Height of the substrate & $1.6 \mathrm{~mm}$ \\
Operating frequency & $2.45 \mathrm{GHz}$ and $5.8 \mathrm{GHz}$ \\
Feeding method & Microstrip line feed \\
Polarization & Linear \\
\hline
\end{tabular}

height $h$, separated by a transmission line of length $L$. Thus, the microstrip is essentially a nonhomogeneous line of two dielectrics - typically, the substrate and the surrounding air. The patch dimensions are calculated by following the simplified formulation of the transmission line model [25].

The width of the antenna is determined to be

$$
W=\frac{V_{0}}{2 f_{r}}\left(\frac{\varepsilon_{r}+1}{2}\right)^{-1 / 2},
$$

where $W, f_{r}, \varepsilon_{r}$, and $V_{0}$ are the width of the patch, the resonant frequency, the dielectric constant of the substrate, and the speed of light in vacuum, respectively.

The effective dielectric constant can be obtained from

$$
\varepsilon_{\mathrm{eff}}=\left(\frac{\varepsilon_{r}+1}{2}\right)+\left(\frac{\varepsilon_{r}-1}{2}\right)\left(1+12 \frac{h}{W}\right)^{-1 / 2},
$$

where $\varepsilon_{\text {eff }}$ is the effective dielectric constant of the substrate.

The fringing effect causes the length of the patch at each end to be extended by distance $\Delta L$ given by

$$
\Delta L=0.412 h \frac{\left(\varepsilon_{\mathrm{eff}}+0.3\right)((W / h)+0.264)}{\left(\varepsilon_{\mathrm{eff}}-0.258\right)((W / h)+0.8)},
$$

where $h$ is the height of the substrate.

The actual length $L$ of the patch is given by

$$
L=\frac{1}{2 f_{r} \sqrt{\varepsilon_{\text {eff }}} \sqrt{\mu_{0} \varepsilon_{0}}}-2 \Delta L,
$$

where $\mu_{0}$ and $\varepsilon_{0}$ are the vacuum permeability and permittivity, respectively.

3.1.3. Feeding Techniques. Antenna performance depends largely on the feeding technique and the location of the optimum feed point. Microstrip line or coaxial probe feeding is widely used in single layer antenna design because these types of feed are easy to design and fabricate. Impedance control in microstrip feeding can be obtained by either edge feeding, inset feeding, or feeding through an additional matching network called a quarter-wave transformer; in this study, microstrip line feeding with an inset feed is used. An inset feed introduces a physical notch that in turn produces junction capacitance. Together, these influence the resonance frequency; as the inset feed point moves inward from the edge, the frequency decreases monotonically, reaching zero at the centre. The input impedance of an inset-fed microstrip 
patch antenna depends mainly on the inset distance (length of the notch) and, to a lesser extent, on the inset width (width of the notch), while the resonant frequency is much more affected by variations in the inset width than in the inset length [26].

The inset distance can be calculated using

$$
R_{50}=R_{\text {inp }} \cos ^{2} \frac{\Pi y_{0}}{L},
$$

where $R_{50}, R_{\text {inp }}$, and $y_{0}$ are the $50 \Omega$ resistance and input impedance of the antenna and the inset distance, respectively.

3.1.4. Ground Plane Dimensions. Although the transmission line model is in theory only applicable to infinite ground planes, for practical considerations, a finite ground plane is used. In order to obtain results similar to those obtained using an infinite ground plane, the size of the ground plane should be greater than the patch dimensions by approximately six to twelve times the substrate thickness. Accordingly, the ground plane dimensions are given by

$$
L_{g}=6 h+L, \quad W_{g}=6 h+W
$$

or

$$
\begin{gathered}
L_{g}=12 h+L, \\
W_{g}=12 h+W,
\end{gathered}
$$

where $L_{g}$ and $W_{g}$ are the length and width of the ground plane, respectively.

For the proposed design, (8) is used to calculate the width of the ground plane, while the length of the ground plane is modified using (9) below to obtain better antenna performance:

$$
L_{g}=12 h+L+L_{f} \text {. }
$$

Here, $L_{f}$ is the length of the feed.

3.1.5. Fractal Iterations. The structure developed in this work consists of a simple fractal structure Sierpinski carpet etched onto a rectangular patch antenna using a conventional rectangular geometry as the generator of the fractal iterations. To build the fractal pattern, the patch is divided into nine smaller congruent rectangles, the central rectangle is then removed, and a similar procedure is followed in subsequent iterations for the remaining rectangles.

As each iteration is added, an area of the radiating patch is removed. The iterative process is based on the following rule:

$$
\begin{gathered}
N_{n}=8^{n}, \quad L_{n}=\left(\frac{1}{3}\right)^{n}, \\
A_{n}=\left(\frac{8}{9}\right)^{n},
\end{gathered}
$$

where $N_{n}$ is the number of rectangles covering the radiating material, $L_{n}$ is the length of the squares removed in each iteration, and $A$ is the area of the radiating patch remaining after each iteration.

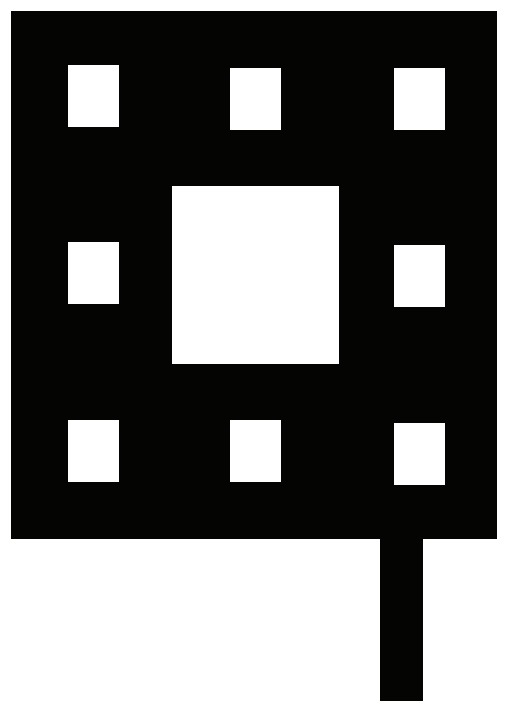

(a)

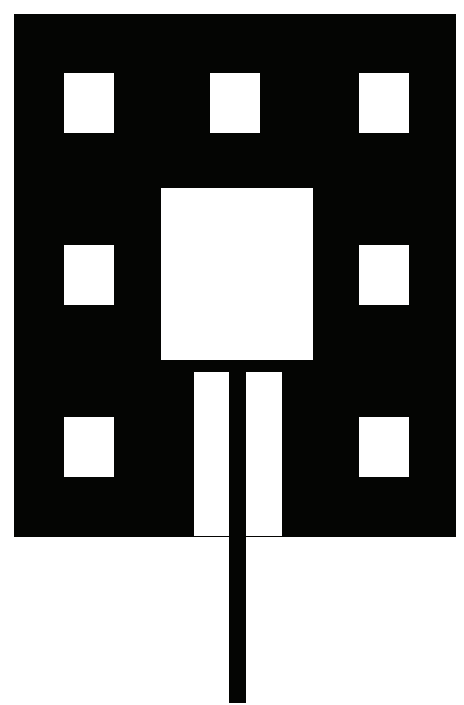

(b)

FIgure 1: (a) Second-order Sierpinski carpet microstrip patch antenna and (b) inset-fed modified Sierpinski carpet microstrip patch antenna.

3.2. Simulation Procedure. The antenna was simulated using the finite-element-method-(FEM-) based Ansoft high frequency structure simulator (HFSS) Ver.10, a high-performance full-wave EM field simulator of arbitrary 3D volumetric passive devices based on a Microsoft Windows graphical user interface. As discussed above, miniaturization of fractal structures involves the process of removing parts from a basic structure; in the proposed design, this removal procedure is performed in an iterative manner.

Iterations of a Sierpinski carpet microstrip patch antenna (SCMPA) performed up to the third order are explained in [4], which shows that antenna performance in terms of reflection loss, impedance matching, and antenna gain remains nearly constant in the first through third iterations. However, while the second iteration experiences the same percentage of size reduction as the third iteration, it is structurally less 


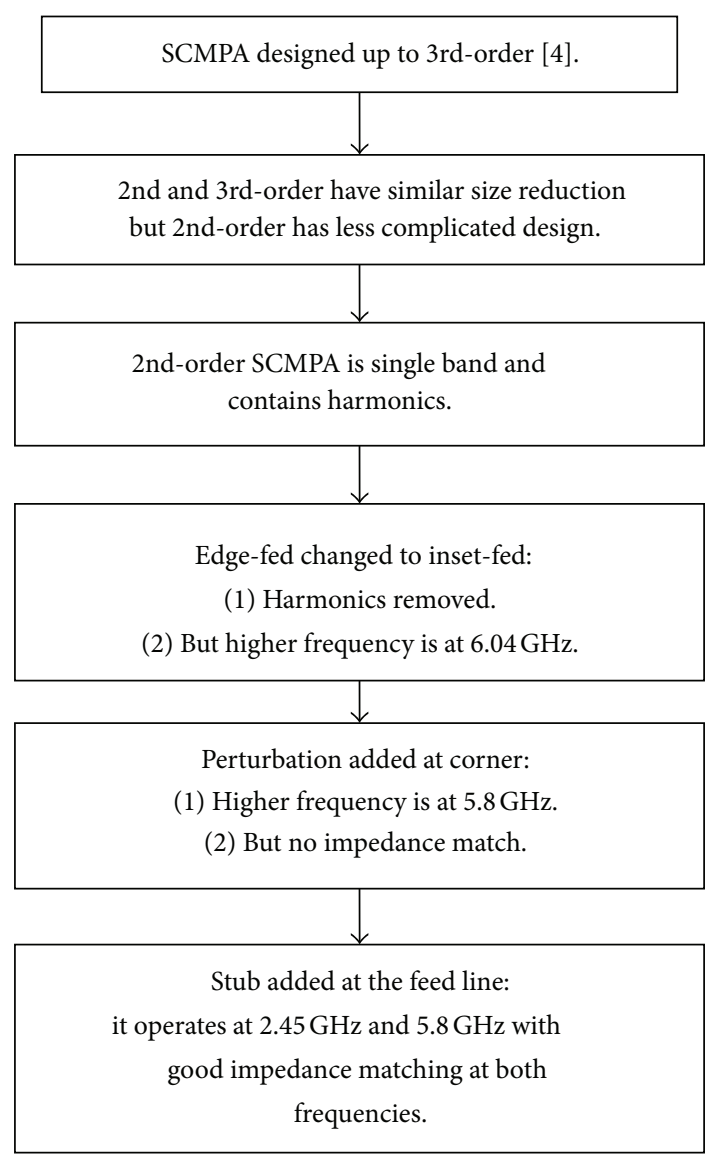

FIGURE 2: Workflow diagram.

complicated [4]. An efficient rectenna with a second-order SCMPA is described in [27]. In the proposed design, the second fractal iteration of a Sierpinski carpet is modified to produce a dual band antenna. The workflow diagram explaining the iterative design approach is shown in Figure 2.

The feeding configuration of the second-order SCMPA changed from edge- to inset-fed is shown in Figures 1(a) and $1(\mathrm{~b})$, respectively, with one of the fractal carpet sections replaced by the inset notch shown in Figure 1(b). By introducing this inset, unwanted harmonics are removed; the return losses of the edge- and inset-fed second-order iterated edgefed Sierpinski fractal antennas are shown in Figure 3.

The return loss of inset-fed antenna shows frequency dips at 2.45 and $6.04 \mathrm{GHz}$, where unwanted harmonics have been removed without using an additional filter component. However, as the higher frequency is still not equivalent to our objective of $5.8 \mathrm{GHz}$, the SCMPA requires further modification in the form of perturbation added to the corner of the patch. Although this changes the higher frequency to $5.8 \mathrm{GHz}$, the impedance match is not improved, and therefore a small stub is added to the feed line. In order to show resonance at 2.45 and $5.8 \mathrm{GHz}$ with fine impedance matching, the simulation procedure required a number of parametric analyses, the results of which are shown in the final dual band miniaturized antenna structure in Figure 4 (with units of length in millimetres). This dual band antenna is $25.98 \%$ smaller than its conventional rectangular patch counterpart.

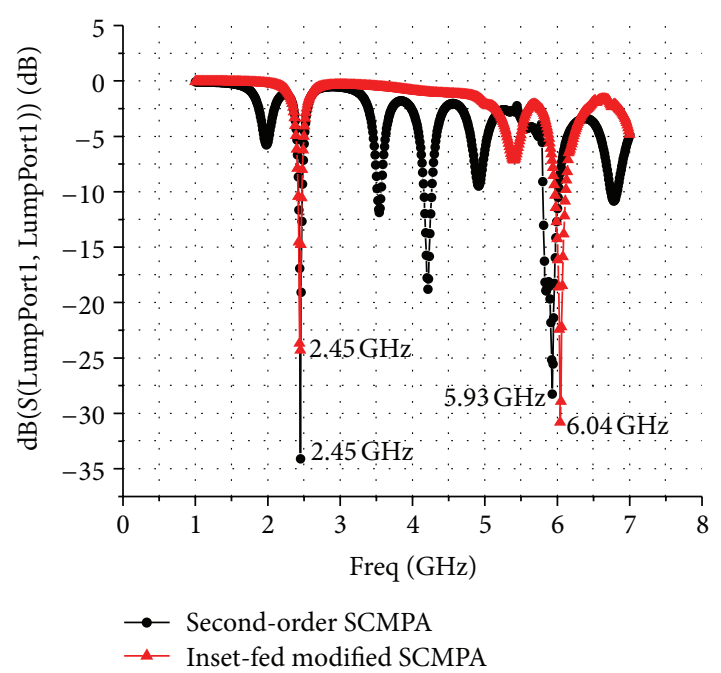

FIGURE 3: Return loss of second-order Sierpinski carpet microstrip patch antenna and inset-fed modified Sierpinski carpet microstrip patch antenna.

\section{Simulation and Measured Results}

The designed dual band antenna through the process above is fabricated on an FR4 substrate (Figure 5). In this section, the performance of the proposed antenna is demonstrated through its simulation and measured result. The simulation result in terms of return loss, Smith Chart, voltage standing wave ratio (VSWR), radiation pattern, and impedance versus frequency curve is exhibited and the measured result in terms of return loss, Smith Chart, and VSWR is illustrated to verify the simulation result.

Measurements of return loss, Smith Chart parameters, and VSWR for fabricated antenna were carried out using an Agilent Network Analyser, N5230A. SubMiniature version A (SMA) connectors were used to interface with the network analyser.

The simulated and measured return loss $\left(\mathrm{S}_{11}\right.$ versus frequency) is shown in Figure 6. The simulated antenna resonates at 2.45 and $5.8 \mathrm{GHz}$, with respective return losses of 35.52 and $39.6 \mathrm{~dB}$ and impedance bandwidths of 60 and $200 \mathrm{MHz}$, respectively. The fabricated antenna resonates at 2.45 and $5.76 \mathrm{GHz}$ with respective return losses of 21.2 and $18.22 \mathrm{~dB}$ and impedance bandwidths of 50 and $120 \mathrm{MHz}$, respectively. The percentage bandwidth of an antenna is defined by

$$
\text { Bandwidth }=\frac{f_{\max }-f_{\min }}{f_{r}} \times 100 \%,
$$

where $f_{r}$ is the resonance frequency, and $f_{\max }$ and $f_{\min }$ are determined at $-10 \mathrm{~dB}$. At 2.45 and $5.8 \mathrm{GHz}$, the simulated bandwidth percentages of the dual band modified SCMPA are $2.44 \%$ and $3.44 \%$, respectively. The measured bandwidth percentages at 2.45 and $5.76 \mathrm{GHz}$ are, respectively, $2.04 \%$ and $2.08 \%$.

To enable an efficient transfer of energy, the impedance of the antenna must match that of the transmission cable. In antenna design, the basic objective is typically to obtain 


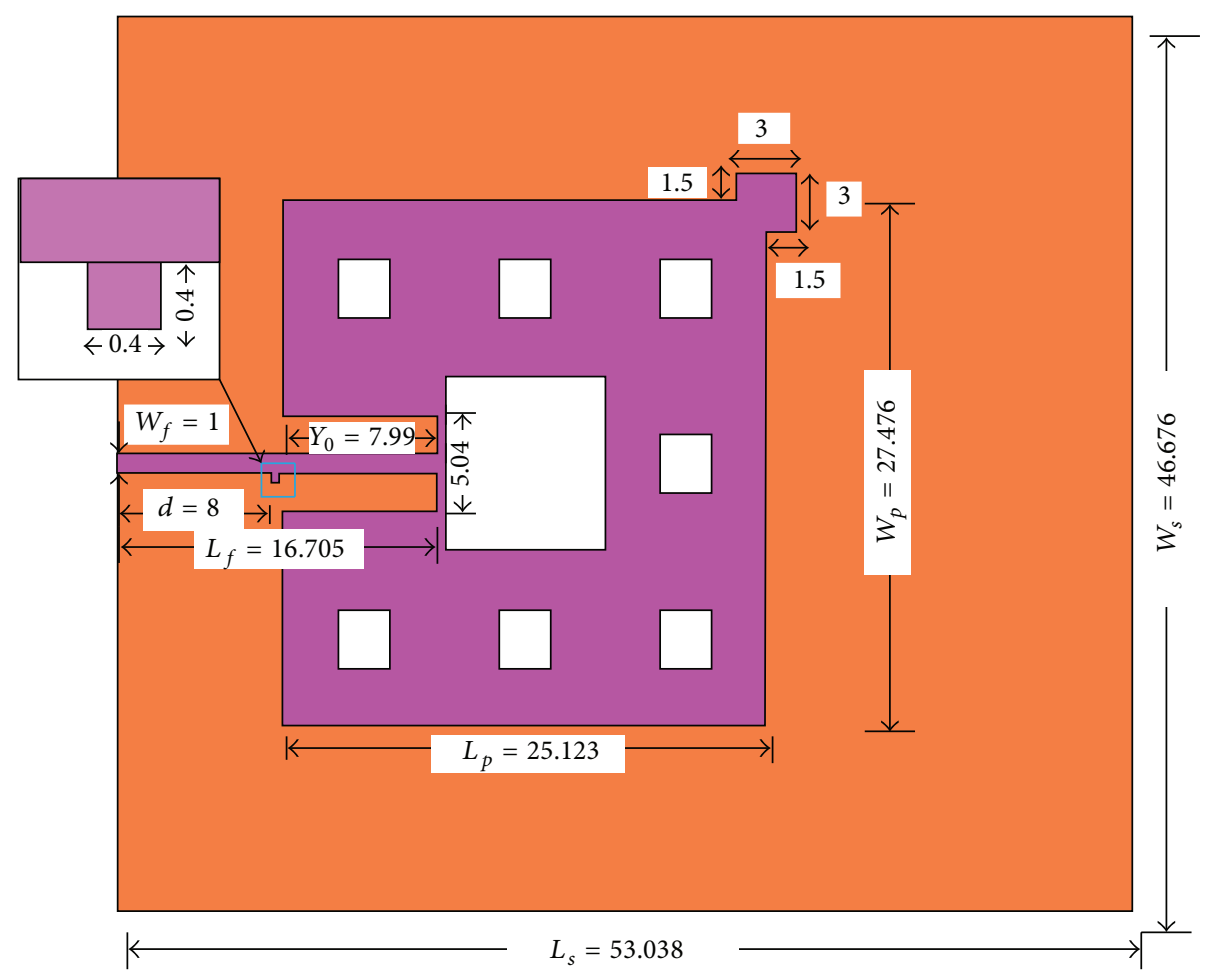

Figure 4: Dual band fractal antenna.

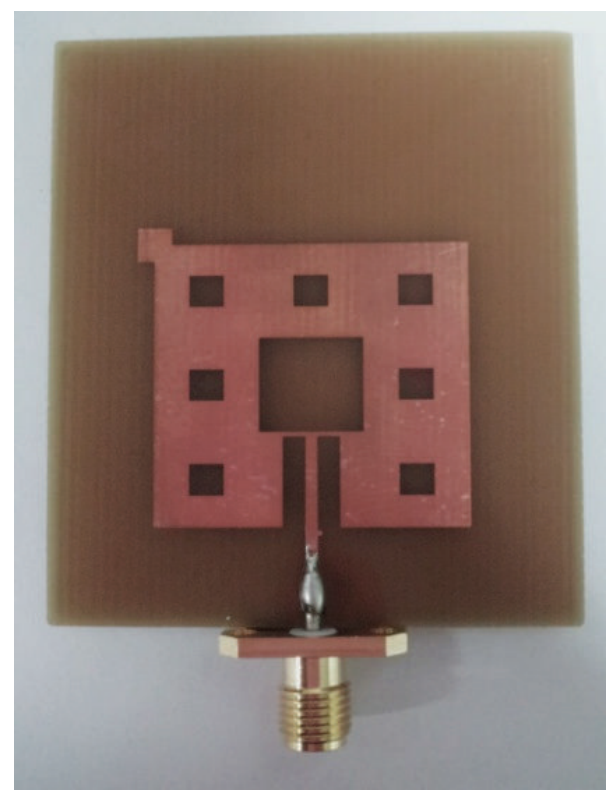

FIGURE 5: Fabricated dual band fractal antenna.

transmission line impedance of $50 \Omega$ in order to match that of the antenna; in the case of mismatch, an impedance matching circuit is required. Figure 7 shows a Smith Chart plotting the reflection coefficient that shows the simulated scattering parameter of the designed antenna at 2.45 and $5.8 \mathrm{GHz}$. Because the reflection coefficient corresponds directly to impedance, this Chart is equivalent to a plot of the impedance; 2.45 and $5.8 \mathrm{GHz}$ can be found at the centre of

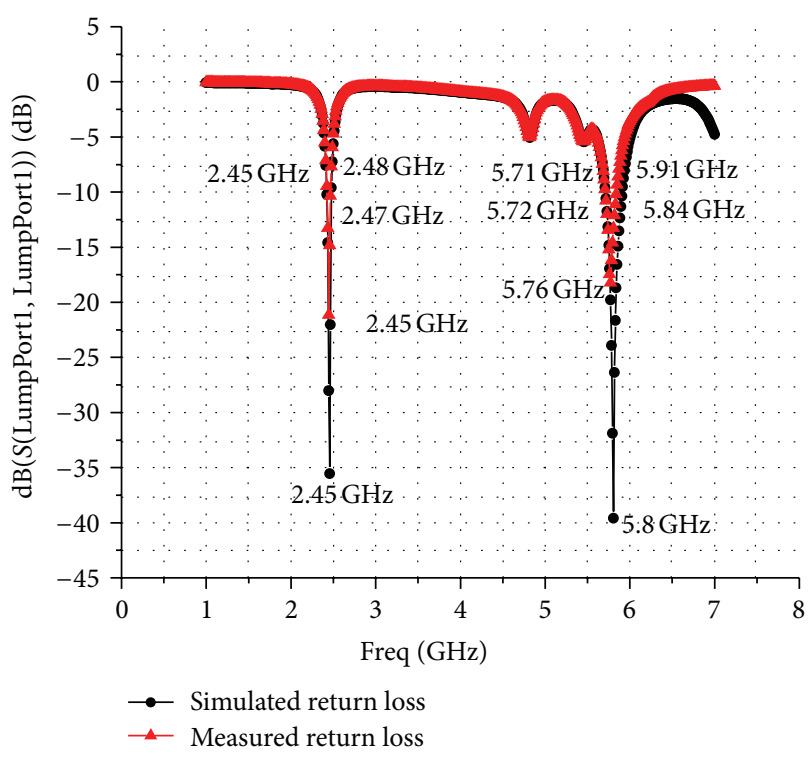

FIGURE 6: Simulated and measured return loss of dual band fractal antenna.

the Chart, indicating that, at these frequencies, the antenna has zero reflection coefficients and is perfectly matched. The Smith Chart obtained through fabricated antenna is shown in Figure 8.

Figure 9 shows the simulated impedance versus frequency curve. The real component of impedance $(Z)$ is nearly equal to $50 \Omega$ and an imaginary component of around $0^{\circ}$ at both frequencies. Together, Figures 7 and 9 show that good 


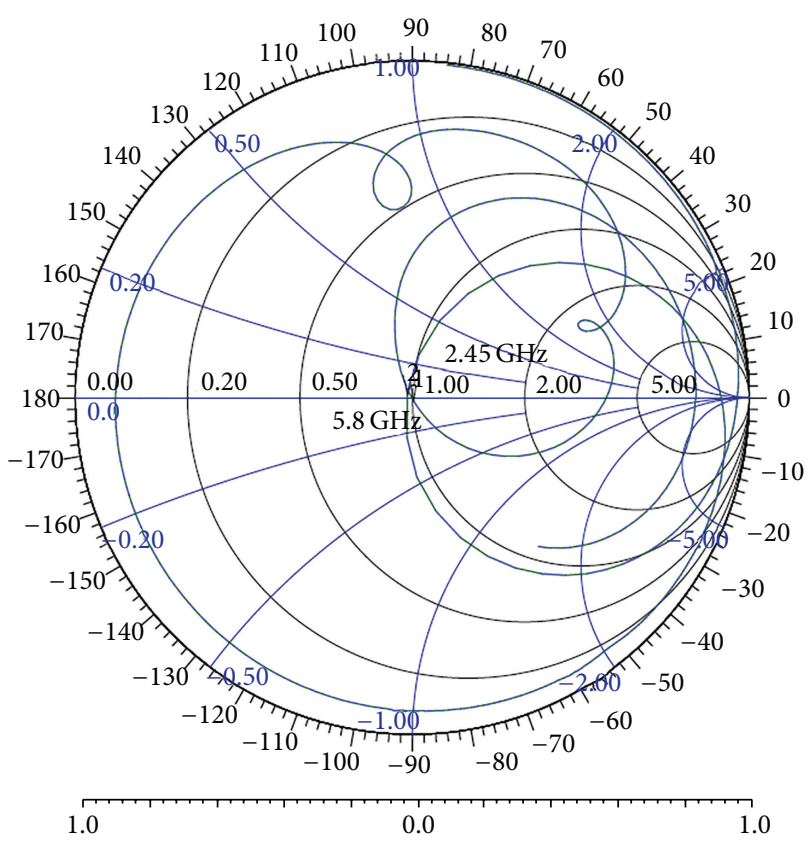

FIGURE 7: Simulated Smith Chart of dual band fractal antenna.

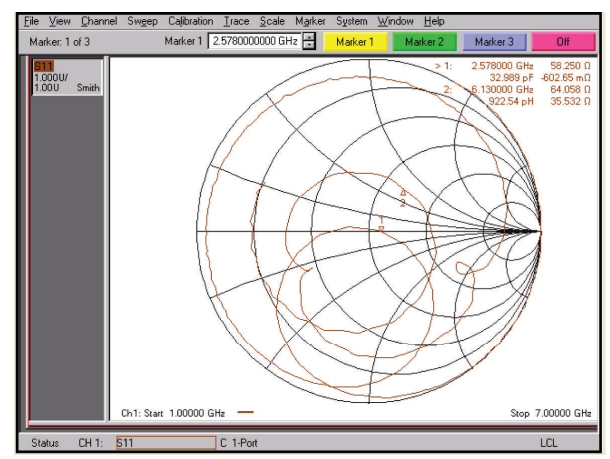

FIGURE 8: Measured Smith Chart of dual band fractal antenna.

impedance matching is obtained in both the frequency bands even when the size is reduced through fractal geometry.

The VSWR characteristics of the designed antenna fall into the range of 1-2 for both simulated and fabricated antenna; from Figure 10, these can be seen to be 1.03 and 1.02 at 2.45 and $5.8 \mathrm{GHz}$, respectively, for simulated antenna and 1.108 and 1.191 at $2.453 \mathrm{GHz}$ and $5.78 \mathrm{GHz}$, respectively, for fabricated antenna.

The far-field simulated radiation patterns at the respective resonance frequencies are given in Figures 11(a) and 11(b), which show maximum simulated gains of 2.7 and $2.7 \mathrm{~dB}$ at 2.45 and $5.8 \mathrm{GHz}$, respectively. The presented radiation pattern corresponds to linear polarization. The red curve in the radiation pattern is for phi $=0^{\circ}$ which is $E$ plane $(X Z$ plane) and the blue curve is for phi $=90^{\circ}$ which is $H$ plane ( $Y Z$ plane).

A comparison between the simulated and measured outputs is provided in Table 2. The measured higher frequency is shifted slightly in frequency versus the S11 curve from the simulation; however, the measured lower frequency is exactly

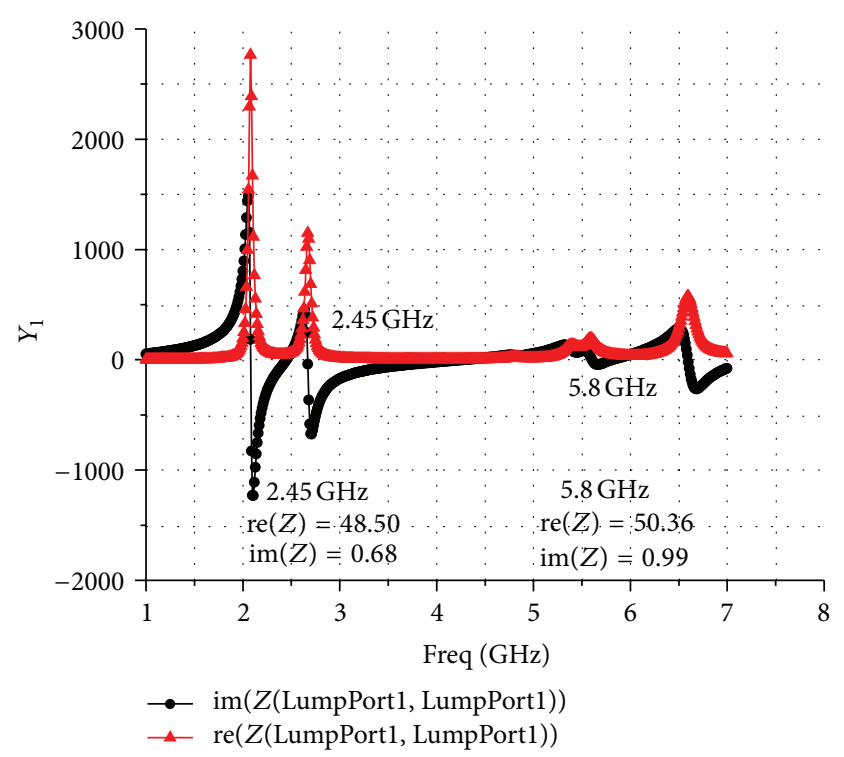

FIGURE 9: Impedance versus frequency curve of dual band fractal antenna.

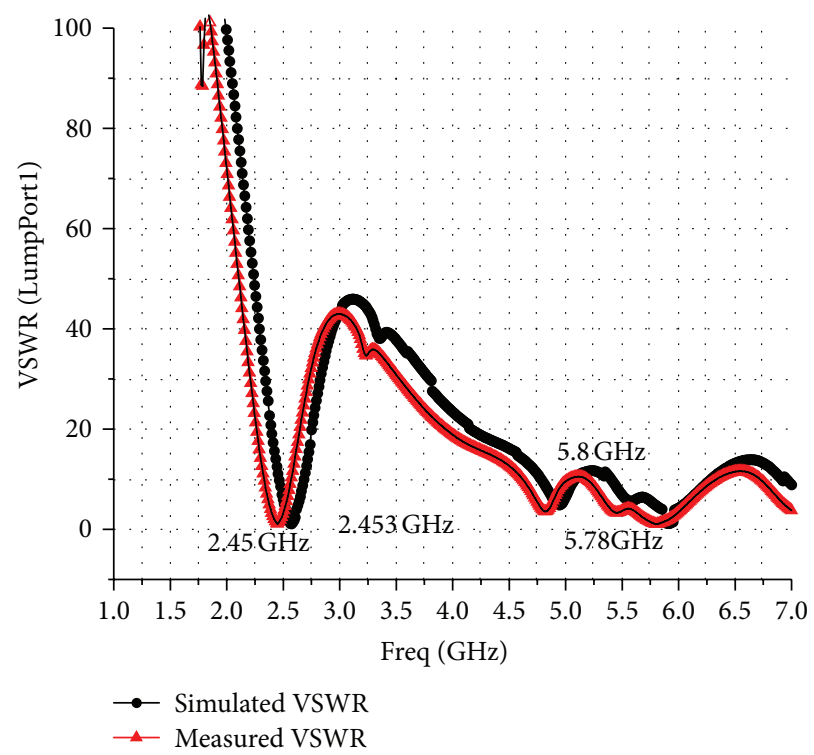

FIGURE 10: Simulated and measured VSWR of dual band fractal antenna.

2.45 GHz. The measured impedance bandwidth and bandwidth percentage are also similar to the simulation results. In the Smith Chart, the measured resonant frequencies are shifted to 2.47 and $5.73 \mathrm{GHz}$, as shown in Figure 8. The difference seen in between simulated and measured Smith Chart is because of the measurement carried out in the open environment and environmental effects have degraded the performance. If the measurement is carried out in anechoic chamber, then better performance can be obtained. The deviations are also partly attributed to inaccuracies in the antenna manufacturing process. As shown in Figure 10, the measured frequency in the VSWR graph is slightly shifted; nevertheless, its value is still between 1 and 2 . Thus, the 

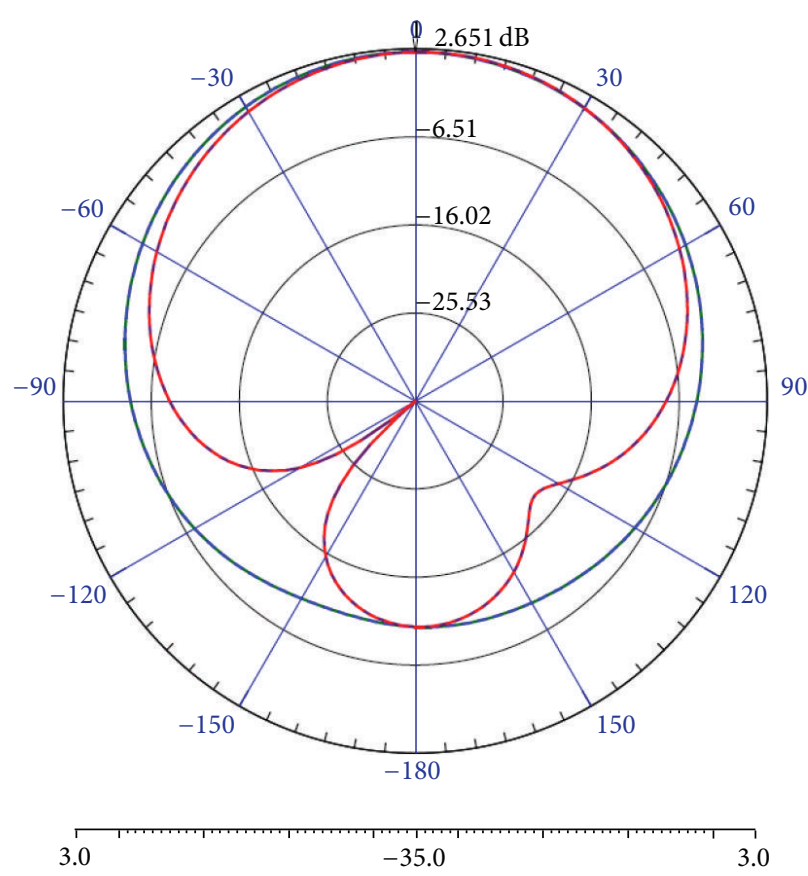

(a)

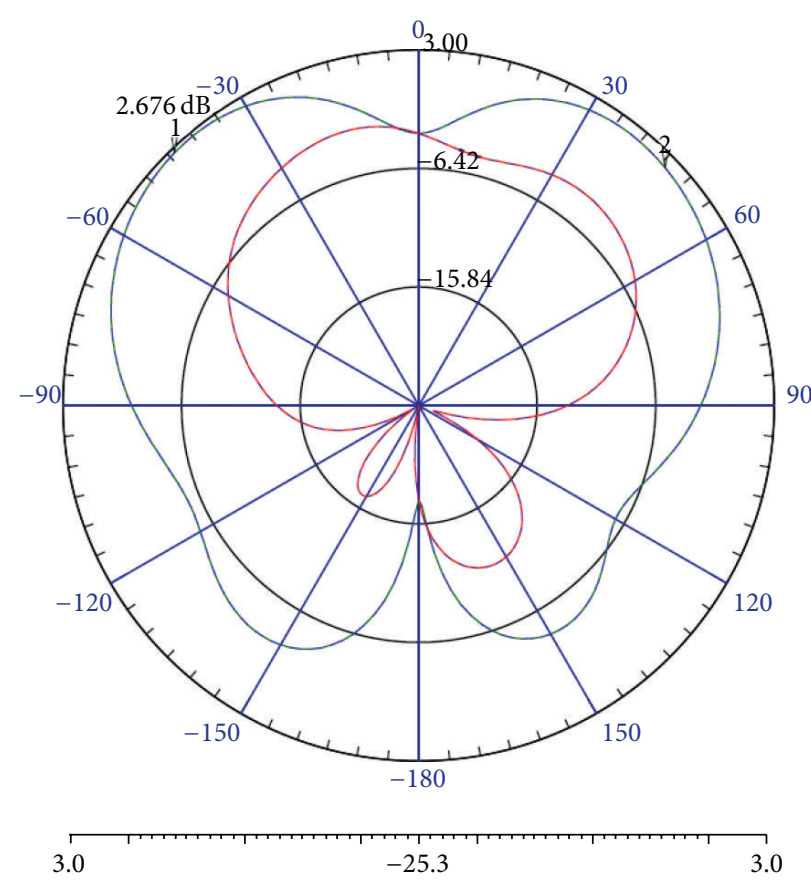

(b)

FIGURE 11: Simulated radiation pattern of dual band fractal antenna (a) at $2.45 \mathrm{GHz}$ (b) at $5.8 \mathrm{GHz}$.

TABLE 2: Comparison of simulated and measured result of dual band antenna.

\begin{tabular}{|c|c|c|c|c|}
\hline \multirow[b]{2}{*}{ Resonant frequency } & \multicolumn{2}{|c|}{ Simulated } & \multicolumn{2}{|c|}{ Measured } \\
\hline & $2.45 \mathrm{GHz}$ & $5.8 \mathrm{GHz}$ & $2.45 \mathrm{GHz}$ & $5.76 \mathrm{GHz}$ \\
\hline Return loss & $35.5 \mathrm{~dB}$ & $39.6 \mathrm{~dB}$ & $21.2 \mathrm{~dB}$ & $18.2 \mathrm{~dB}$ \\
\hline $\begin{array}{l}\text { Impedance } \\
\text { bandwidth }\end{array}$ & $60 \mathrm{MHz}$ & $200 \mathrm{MHz}$ & $50 \mathrm{MHz}$ & $120 \mathrm{MHz}$ \\
\hline $\begin{array}{l}\text { Bandwidth } \\
\text { percentage }\end{array}$ & $2.44 \%$ & $3.44 \%$ & $2.04 \%$ & $2.08 \%$ \\
\hline Resonant frequency & $2.45 \mathrm{GHz}$ & $5.8 \mathrm{GHz}$ & $2.453 \mathrm{GHz}$ & $5.78 \mathrm{GHz}$ \\
\hline VSWR & 1.03 & 1.02 & 1.108 & 1.191 \\
\hline
\end{tabular}

deviations observed between the simulated and the measured results are minor and can be attributed to environmental effects and antenna manufacturing process.

\section{Conclusion}

This study produced a modified Sierpinski fractal-based microstrip patch antenna for a rectenna with a significantly reduced size that can operate at two frequencies, 2.45 and $5.8 \mathrm{GHz}$. Owing to the space-filling nature of fractal geometry, a second-order fractal iteration effectively reduced the size of the antenna, while fractal self-similarity was used to generate two resonating frequencies, although the higher frequency of the initial design was not at the desired $5.8 \mathrm{GHz}$. To correct this, perturbation was added to shift the higher frequency resonance to $5.8 \mathrm{GHz}$, and a stub was added to the feed line to obtain fine impedance matching at both frequencies. The designed antenna has a simulated return loss greater than $35 \mathrm{~dB}$ and good impedance matching at both frequencies. Simulated gains of 2.7 and $2.7 \mathrm{~dB}$ were attained at 2.45 and $5.8 \mathrm{GHz}$, respectively, and an overall antenna size reduction of $25.98 \%$ was obtained. The dual band antenna was then fabricated on an FR4 substrate to verify simulation results. Although deviations between fabricated and simulated results occurred owing to environmental conditions and manufacturing inaccuracies, these were slight and, on the basis of the overall results, it can be concluded that applying fractal geometry to a patch antenna is a simple and effective technique for developing multiband antennas of minimal size. To obtain higher gain, the proposed design can be etched onto other substrates with lower permittivity, such as Teflon. Furthermore, an arrayed fractal antenna can be designed to increase overall gain, and a stacked Sierpinski carpet fractal antenna can be designed to obtain a wider bandwidth than a single antenna. Such antennas can be integrated with a rectifier circuit generating rectennas in order to harvest RF energy at dual frequencies.

\section{Conflict of Interests}

The authors declare that there is no conflict of interests regarding the publication of this paper.

\section{Acknowledgments}

This research was supported by Basic Science Research Program through the National Research Foundation of Korea (NRF) funded by the Ministry of Education (NRF-20090093828). And this research was supported partially by the MSIP (Ministry of Science, ICT and Future Planning), Korea, 
under the C-ITRC (Convergence Information Technology Research Centre) Support Program (NIPA-2013-H0401-132006) supervised by the NIPA (National IT Industry Promotion Agency).

\section{References}

[1] S. Shrestha, S.-K. Noh, and D. Y. Choi, "Comparative study of antenna designs for RF energy harvesting," International Journal of Antennas and Propagation, vol. 2013, Article ID 385260, 10 pages, 2013.

[2] J. P. Gianvittorio and Y. Rahmat-Samii, "Fractal antennas: a novel antenna miniaturization technique, and applications," IEEE Antennas and Propagation Magazine, vol. 44, no. 1, pp. 20 36, 2002.

[3] J. Anguera, E. Martínez, C. Puente, C. Borja, and J. Soler, "Broad-band dual-frequency microstrip patch antenna with modified Sierpinski fractal geometry," IEEE Transactions on Antennas and Propagation, vol. 52, no. 1, pp. 66-73, 2004.

[4] S. Shrestha, J. J. Park, S. K. Noh, and D. Y. Choi, "Design of 2.45 GHz sierpinski fractal based miniaturized patch antenna," in Proceedings of the 18th Asia-Pacific Conference on Communication (APCC '12), pp. 36-41, October 2012.

[5] J. K. Ali, "A new reduced size multiband patch antenna structure based on Minkowski pre-fractal geometry," Journal of Engineering and Applied Sciences, vol. 2, pp. 1120-1124, 2007.

[6] C. Borja and J. Romeu, "On the behavior of Koch island fractal boundary microstrip patch antenna," IEEE Transactions on Antennas and Propagation, vol. 51, no. 6, pp. 1281-1291, 2003.

[7] T. K.-C. Lo and Y. Hwang, "Microstrip antennas of very high permittivity for personal communications," in Proceedings of the Asia-Pacific Microwave Conference (APMC '97), vol. 1, pp. 253256, December 1997.

[8] N. G. Alexopoulos and D. R. Jackson, "Fundamental superstrate (cover) effects on printed circuit antennas," IEEE Transactions on Antennas and Propagation, vol. 32, no. 8, pp. 807-816, 1984.

[9] T.-C. Yo, C.-M. Lee, C.-M. Hsu, and C.-H. Luo, "Compact circularly polarized rectenna with unbalanced circular slots," IEEE Transactions on Antennas and Propagation, vol. 56, no. 3, pp. 882-886, 2008.

[10] G. A. Vera, A. Georgiadis, A. Collado, and S. Via, "Design of a $2.45 \mathrm{GHz}$ rectenna for electromagnetic (EM) energy scavenging," in Proceedings of the IEEE Radio and Wireless Symposium (RWW'10), pp. 61-64, January 2010.

[11] D. T. Notis, P. C. Liakou, and D. P. Chrissoulidis, "Dual polarized microstrip patch antenna, reduced in size by use of peripheral slits," in Proceedings of the 34th European Microwave Conference, vol. 1, pp. 125-128, October 2004.

[12] J. K. Ali, "A new compact size microstrip patch antenna with irregular slots for handheld GPS application," Engineering and Technology, vol. 26, no. 10, 2008.

[13] J.-Y. Park, C. Caloz, Y. Qian, and T. Itoh, "A compact circularly polarized subdivided microstrip patch antenna," IEEE Microwave and Wireless Components Letters, vol. 12, no. 1, pp. 18-19, 2002.

[14] A. Kordzadeh and F. H. Kashani, "A new reduced size microstrip patch antenna with fractial shaped defects," Progress in Electromagnetics Research B, no. 11, pp. 29-37, 2009.

[15] Z.-W. Yu, G.-M. Wang, X.-J. Gao, and K. Lu, "A novel small-size single patch microstrip antenna based on Koch and Sierpinski fractal-shapes," Progress in Electromagnetics Research Letters, vol. 17, pp. 95-103, 2010.

[16] M. Jamshidifar, J. Nourinia, C. Ghobadi, and F. Arazm, "Wideband fractal butterfly patch antenna," Iranian Journal of Electrical and Computer Engineering, vol. 7, no. 2, pp. 134-136, 2008.

[17] Y. Tawk, J. Costantine, and C. G. Christodoulou, "A frequency reconfigurable rotatable microstrip antenna design," in Proceedings of the IEEE International Symposium on Antennas and Propagation (APUSURSI '10), pp. 1-4, July 2010.

[18] N. Singh, D. P. Yadav, S. Singh, and R. K. Sarin, "Compact corner truncated triangular patch antenna for WiMax application," in Proceedings of the 10th Mediterranean Microwave Symposium (MMS '10), pp. 163-165, August 2010.

[19] M. Ali, G. Yang, and R. Dougal, "A new circularly polarized rectenna for wireless power transmission and data communication," IEEE Antennas and Wireless Propagation Letters, vol. 4, no. 1, pp. 205-208, 2005.

[20] K. Chung, Y. Nam, T. Yun, and J. Choi, "Reconfigurable microstrip patch antenna with switchable polarization," ETRI Journal, vol. 28, no. 3, pp. 379-382, 2006.

[21] D. D. Krishna, A. R. Chandran, and C. K. Aanandan, "A compact dual frequency antenna with Sierpinski gasket based slots," in Proceedings of the 10th European Conference on Wireless Technology (ECWT '07), pp. 320-322, October 2007.

[22] D. D. Krishna, M. Gopikrishna, C. K. Anandan, P. Mohanan, and K. Vasudevan, "CPW-fed Koch fractal slot antenna for WLAN/WiMAX applications," IEEE Antennas and Wireless Propagation Letters, vol. 7, pp. 389-392, 2008.

[23] J. Ghalibafan and F. H. Kashani, "A circularly polarized fractal microstrip antenna for RFID applications," in Proceedings of the IEEE International Symposium on Radio-Frequency Integration Technology (RFIT '09), pp. 319-322, January 2009.

[24] N. Bisht and P. Kumar, "A dual band fractal circular microstrip patch antenna for C-band applications," in Progress in Electromagnetics Research Symposium (PIERS '11), pp. 852-855, Suzhou, China, September 2011.

[25] C. A. Balanis, Antenna Theory Analysis and Design, John Wiley \& Sons, New York, NY, USA, 3rd edition, 2005.

[26] M. Ramesh and K. B. Yip, "Design formula for inset fed microstrip patch antenna," Journal of Microwaves and Optoelectronics, vol. 3, no. 3, pp. 5-10, 2003.

[27] D. Y. Choi, S. Shrestha, J. J. Park, and S. K. Noh, "Design and performance of an efficient rectenna incorporating a fractal structure," International Journal of Communication Systems, 2013. 

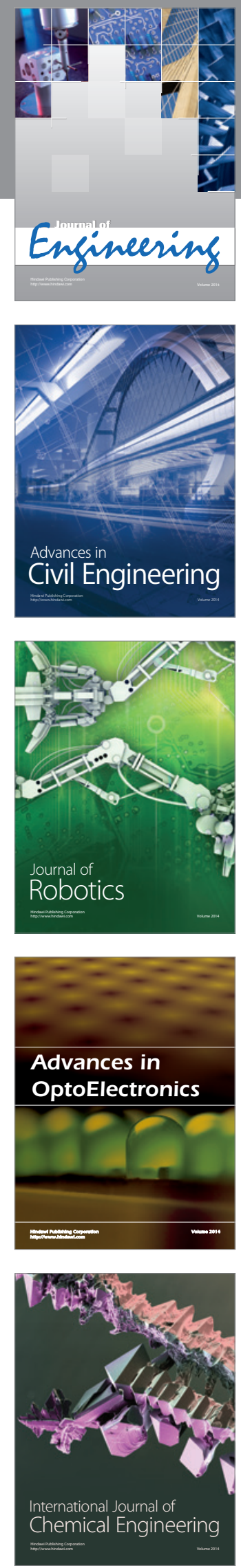

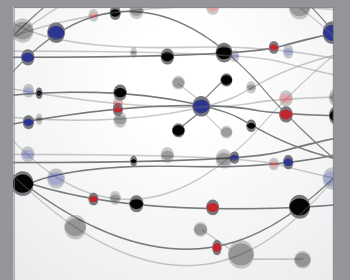

The Scientific World Journal
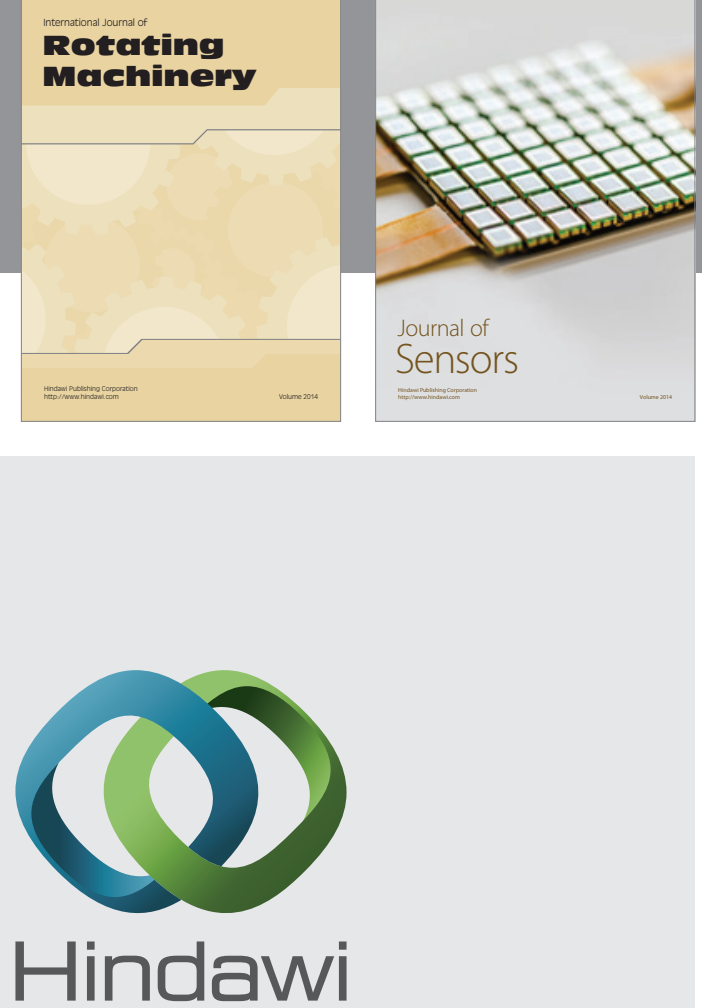

Submit your manuscripts at http://www.hindawi.com
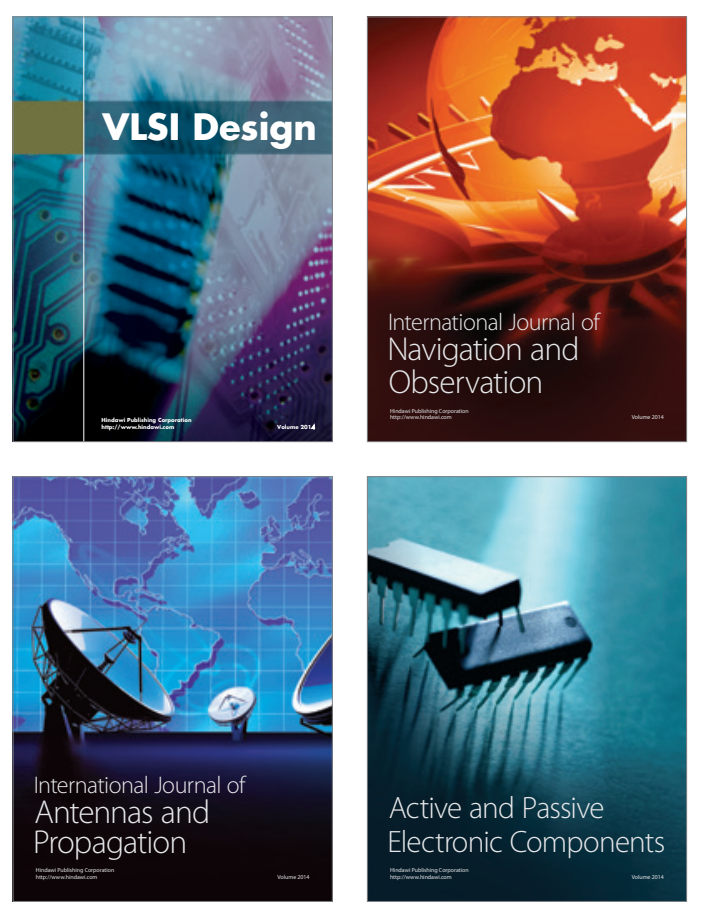
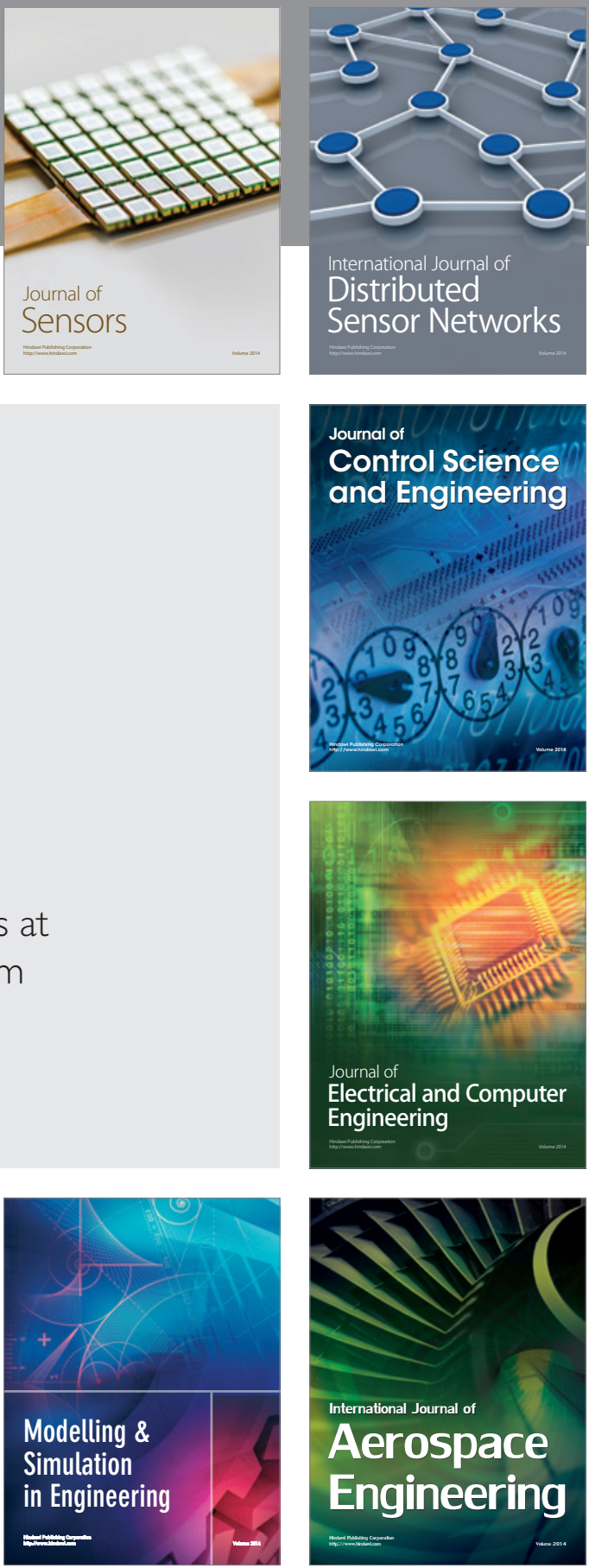

Journal of

Control Science

and Engineering
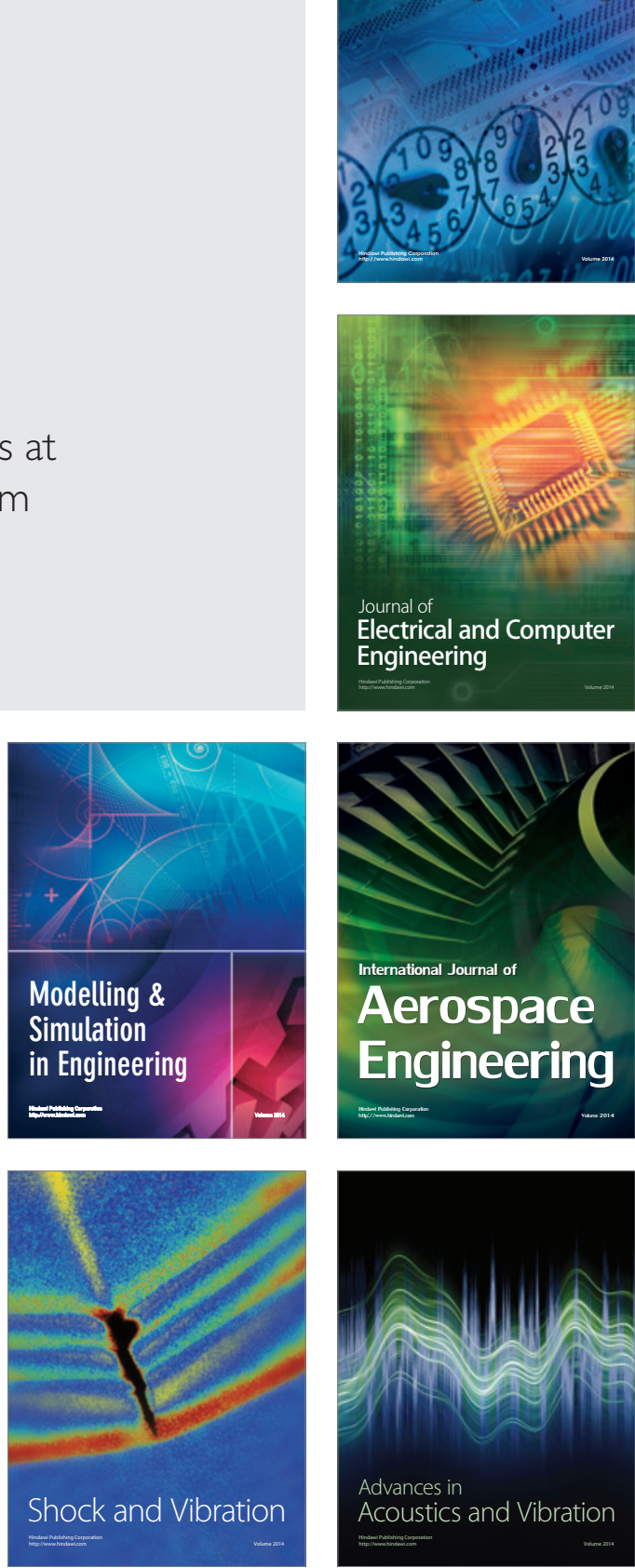